\title{
On the Steadily Rotating Spirals
}

\author{
Jong-Shenq GuO $^{\dagger 1}$, Ken-Ichi NAKAmurA ${ }^{\dagger 2}$, \\ Toshiko OGIWARA ${ }^{\dagger 3}$ and Je-Chiang TsaI ${ }^{\dagger 4 *}$ \\ ${ }^{\dagger 1}$ Department of Mathematics, National Taiwan Normal University, \\ 88, S-4 Ting Chou Road, Taipei 116, Taiwan \\ E-mail:jsguo@math.ntnu.edu.tw \\ $\dagger^{2}$ Department of Computer Science, University of Electro-Communications, \\ Tokyo 182-8585, Japan \\ E-mail: nakamura@im.uec.ac.jp \\ $\dagger 3$ Department of Mathematics, Josai University, \\ Saitama 350-0295, Japan \\ E-mail: toshiko@math.josai.ac.jp \\ ${ }^{\dagger}$ Department of Computer Science, National Taiwan Ocean University, \\ 2, Pei-Ning Road, Keelung 202, Taiwan \\ E-mail: tsaijc@mail.ntou.edu.tw
}

Received February 25, 2005

Revised May 26, 2005

\begin{abstract}
We study an autonomous system of two first order ordinary differential equations. This system arises from a model for steadily rotating spiral waves in excitable media. The sharply located spiral wave fronts are modeled as planar curves. Their normal velocity is assumed to depend affine linearly on curvature. The spiral tip rotates along a circle with a constant positive rotation frequency. The tip neither grows nor retracts tangentially to the curve. With rotation frequency as a parameter, we obtain the complete classification of solutions of this system. Besides providing another approach to derive the results obtained by Fiedler-Guo-Tsai for spirals with positive curvature, we also obtain many more different solutions. In particular, we obtain spiral wave solutions with sign-changing curvature and with negative curvature.
\end{abstract}

Key words: steadily rotating spiral wave, spiral wave solution, phase plane

\section{Introduction}

Rotating spiral wave patterns are commonly observed in many spatio-temporal evolution of excitable systems, such as Belousov-Zhabotinsky system, FitzHughNagumo system, etc. These patterns are usually modeled by a sharp transition layer in the related reaction-diffusion systems. In order to simplify the analysis of this pattern, instead of using the reaction-diffusion system, one usually uses the so-called kinematic theory of spiral waves. That is the sharply located spiral wave fronts are modeled as a family of planar curves. For more physical background and mathematical theory on the spiral waves, we refer the reader to the survey papers of Meron [8] and Tyson-Keener [11] and the references therein. See also recent papers by Keener [7], Mikhailov-Davydov-Zykov [9], Brazhnik [1], Ikoda-IshimuraYamaguchi [6], Ogiwara-Nakamura [10], Fiedler-Guo-Tsai [2, 3], Guo-Lo-Tsai [5], and Guo-Ishimura-Wu [4].

\footnotetext{
${ }^{*}$ To whom the correspondence should be addressed.
} 
In the kinematic theory of spiral waves, the sharply fronts are represented by a family of curves in $\mathbf{R}^{2}$ parametrized by $t \geq 0$ such that each curve has one free tip. We shall always choose the normal vector to be the left-hand normal to the tangent vector. Also, we choose the curvature to be positive when the curve is winding in the clockwise direction.

Let $s$ be the arc length measured from the tip (so that the tip is corresponding to $s=0), \kappa:=\kappa(s, t)$ be the curvature, $u:=u(s, t)$ be the normal velocity, and $G:=G(t)$ be the tangential velocity at the tip. Then the following equation can be derived from the definitions of the normal and tangent vectors, normal and tangential velocities, and the Frenet-Serret Theorem in the plane (see, for example, $[4])$ :

$$
\kappa_{t}+u_{s s}+\left(\kappa \int_{0}^{s} \kappa u d \xi\right)_{s}+G \kappa_{s}=0, s \geq 0, t \geq 0 .
$$

In the study of steadily rotating spiral wave in the kinematic theory of excitable media (cf. $[1,7,8,9]$ ), we assume that the family of curves keep the same shape for all $t$ and rotate along a core circle in the counterclockwise direction with a constant positive speed such that the tip of the curve neither grows nor retracts in the tangential direction (i.e., $G \equiv 0$ ). Then (1.1) is reduced to

$$
u^{\prime \prime}(s)+\left(\kappa(s) \int_{0}^{s} \kappa(\xi) u(\xi) d \xi\right)^{\prime}=0, \quad s \geq 0
$$

Note that by assumption the position vector is always perpendicular to the normal vector at the tip.

By integrating (1.2) once, we obtain that $u$ and $\kappa$ satisfy the equation

$$
u^{\prime}(s)+\kappa(s) \int_{0}^{s} \kappa(\xi) u(\xi) d \xi=\omega
$$

where $\omega$ is the positive constant angular frequency of the wave. It is also important to note that the radius of the core circle is given by $\rho=|u(0)| / \omega$ with the tip tangent pointing inward to the center of the core circle if $u(0)<0$; outward to the center if $u(0)>0$ (cf. [3]).

Now set

$$
v(s):=\int_{0}^{s} \kappa(\xi) u(\xi) d \xi, \quad s>0 .
$$

Then (1.3) is reduced to the following system determining the desired relationship between the function $\kappa(s), u(s)$ and $v(s)$ :

$$
\begin{aligned}
& \frac{d v}{d s}=\kappa u, \\
& \frac{d u}{d s}=\omega-\kappa v .
\end{aligned}
$$


If we assume the normal velocity $u$ of the curve depends on the local curvature $\kappa$ according to the following relation

$$
u=c-D \kappa,
$$

then (1.5)-(1.6) is reduced to the following system:

$$
\begin{aligned}
& \frac{d v}{d s}=\kappa(c-D \kappa), \\
& \frac{d \kappa}{d s}=(\kappa v-\omega) / D .
\end{aligned}
$$

The initial condition for (1.8)-(1.9) shall be given by

$$
v(0)=0, \quad \kappa(0)=\kappa_{0},
$$

where $\kappa_{0}$ is the curvature at the tip.

Following [12], to obtain the dimensionless form, we introduce the following change of variables:

$$
\tilde{\kappa}=(D / c) \kappa, \quad \tilde{v}=v / c, \quad \tilde{s}=(c / D) s, \quad \tilde{\omega}=\left(D / c^{2}\right) \omega .
$$

In these variables the problem (1.8)-(1.10) can be reduced to the following problem $\left(P_{\tilde{\omega}}\right)$ :

$$
\begin{aligned}
& \frac{d \tilde{v}}{d \tilde{s}}=\tilde{\kappa}(1-\tilde{\kappa}), \\
& \frac{d \tilde{\kappa}}{d \tilde{s}}=\tilde{v} \tilde{\kappa}-\tilde{\omega} \\
& \tilde{v}(0)=0, \quad \tilde{\kappa}(0)=b,
\end{aligned}
$$

where $b=(D / c) \kappa_{0}$. The local existence and uniqueness of solutions of $\left(P_{\tilde{\omega}}\right)$ are trivial.

Hereafter for notational convenience we shall suppress the tilde by assuming that $c=D=1$. In the sequel, we denote $(v(s ; \omega, b), \kappa(s ; \omega, b))$ the solution of $\left(P_{\omega}\right)$ to specify the dependence of $(v, \kappa)$ on the parameters $\omega$ and/or $b$.

In [6], they studied the equations (1.3) and (1.7) with the initial condition

$$
\kappa(0)=\kappa_{0}, \quad \kappa^{\prime}(0)=0 .
$$

They obtained many interesting results for any $\omega \in(-\infty, \infty)$. In particular, for $\omega=0$ the solution is periodic if $\kappa_{0} \in(0,2) \backslash\{1\}$; a constant if $\kappa_{0}=1$; and is monotone decreasing with $\kappa(s) \rightarrow 0$ as $s \rightarrow \infty$ if $\kappa_{0} \geq 2$. In [5], we studied a simplified equation of (1.3), namely,

$$
-\kappa^{\prime}(y)+\kappa(y) \int_{0}^{y} \kappa(\xi) d \xi=\omega
$$


and obtained a family of steadily rotating spiral waves. In [2], we prove that there is a critical value $\bar{\omega}>0$ such that a spiral wave solution $(v, \kappa)$ of $(1.8)-(1.10)$ with $\kappa>0$ on $[0,+\infty)$ exists if and only if $\omega \in(0, \bar{\omega}]$. Moreover, we are able to count the exact number of such spiral wave solutions for any given $\omega \in(0, \bar{\omega}]$. In a companion paper [3], we present an alternative approach via center manifolds and study the Archimedean shape of the rotating spirals.

In this paper, we shall present some new results on the kinematic equation (1.3) with normal-curvature relation (1.7) for steadily rotating spiral waves by studying the associated problem $\left(P_{\omega}\right)$ for $\omega>0$. We obtain the complete classification of solutions of this system. Besides providing another approach to derive the results obtained in $[2,3]$ for spirals with positive curvature, we also obtain many more different solutions. In particular, we obtain spiral wave solutions with sign-changing curvature and with negative curvature.

This paper is organized as follows. In $\S 2$, we first give some preliminary results. In $\S 3$, we shall use another approach to reproduce the result in [2], i.e. we prove that there exists $\bar{\omega}>0$ such that there is a global solution $(v, \kappa)$ of $\left(P_{\omega}\right)$ with $\kappa>0$ on $[0,+\infty)$ if and only if $\omega \in(0, \bar{\omega}]$. Moreover, we are able to count the exact number of such spiral wave solutions for any given $\omega \in(0, \bar{\omega}]$. In $\S 4$, we shall use the tip curvature $b$ as a parameter to classify the solution of $\left(P_{\omega}\right)$. Some geometric explanations shall be given in $\S 5$.

\section{Preliminary}

For $\omega>0$, we define

$$
\begin{aligned}
& \mathcal{D}_{1}=\{(v, \kappa) \mid v>0,0<\kappa<1, v \kappa-\omega>0\}, \\
& \mathcal{D}_{2}=\{(v, \kappa) \mid v>0, \kappa>1, v \kappa-\omega>0\}, \\
& \mathcal{D}_{3}=\{(v, \kappa) \mid \kappa>1, v \kappa-\omega<0\} \\
& \mathcal{D}_{4}=\{(v, \kappa) \mid 0<\kappa<1, v \kappa-\omega<0\} \\
& \mathcal{B}_{1}=\{(v, \kappa) \mid \kappa<0, v \kappa-\omega<0\} \\
& \mathcal{B}_{2}=\{(v, \kappa) \mid v<0, \kappa<0, v \kappa-\omega>0\} .
\end{aligned}
$$

The next lemma follows easily from the phase plane analysis for the following system:

$$
\begin{aligned}
& \frac{d v}{d s}=\kappa(1-\kappa), \\
& \frac{d \kappa}{d s}=v \kappa-\omega .
\end{aligned}
$$

LEMMA 2.1. Let $\omega>0$. The following statements hold.

(1) $v^{\prime}>0, \kappa^{\prime}>0$ for $(v, \kappa) \in \mathcal{D}_{1}$ and $v^{\prime}<0, \kappa^{\prime}>0$ for $(v, \kappa) \in \mathcal{D}_{2}$.

(2) $v^{\prime}<0, \kappa^{\prime}<0$ for $(v, \kappa) \in \mathcal{D}_{3}$ and $v^{\prime}>0, \kappa^{\prime}<0$ for $(v, \kappa) \in \mathcal{D}_{4}$.

(3) $v^{\prime}<0, \kappa^{\prime}<0$ for $(v, \kappa) \in \mathcal{B}_{1}$ and $v^{\prime}<0, \kappa^{\prime}>0$ for $(v, \kappa) \in \mathcal{B}_{2}$. 
(4) $\mathcal{B}_{2}$ and $\{(v, \kappa) \mid \kappa<0\}$ are positively invariant regions for the system (2.1)$(2.2)$.

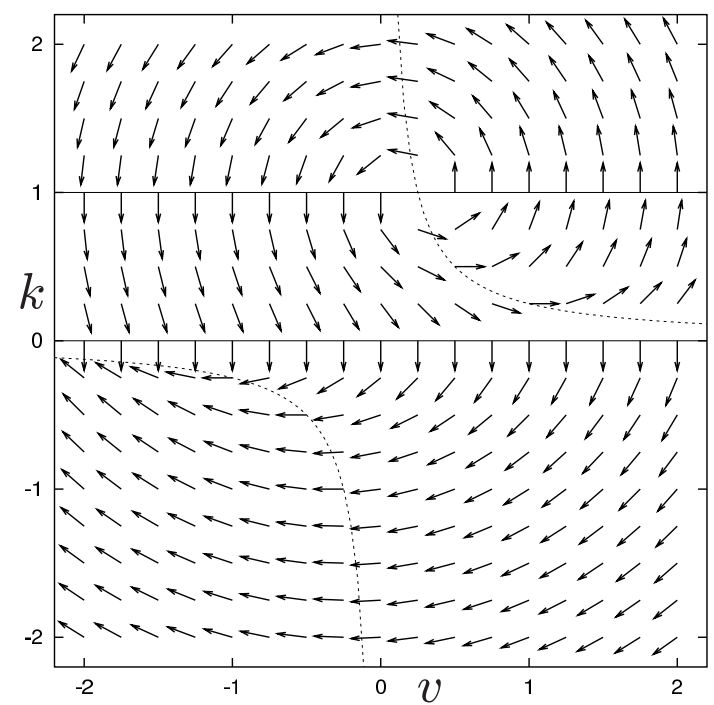

Fig. 1. The (normalized) vector field generated by (2.1)-(2.2) for $\omega=0.250$. The solid lines denote the set $\{d v / d s=0\}$ and the dashed curves denote the set $\{d \kappa / d s=0\}$.

For any $\omega>0,(\omega, 1)$ is the only equilibrium point of $(2.1)-(2.2)$. Since $(\omega, 1)$ is a spiral source for $\omega \in(0,2)$, it is convenient to use the new variables $(r, \theta)$ defined by

$$
v=\omega+r \cos \theta, \quad \kappa=1+r \sin \theta .
$$

Then the system (2.1)-(2.2) becomes

$$
\begin{aligned}
& \frac{d r}{d s}=\omega r \sin ^{2} \theta \\
& \frac{d \theta}{d s}=1+\omega \sin \theta \cos \theta+r \sin \theta .
\end{aligned}
$$

Since $r^{\prime}(s) \leq \omega r(s)$, we see that $r(s)$ is finite (hence both $v(s)$ and $\kappa(s)$ are finite) if $s$ is finite. This and the strict monotonicity of $r(s)$ imply that all solutions of (2.1)(2.2) are defined for all $s \in \mathbf{R}$ and that $\lim _{s \rightarrow-\infty} r(s)=0, \lim _{s \rightarrow+\infty} r(s)=+\infty$.

Lemma 2.2. Let $(v, \kappa)$ be the solution of (2.1)-(2.2) with the initial value $(d, 0)$ for $d \in \mathbf{R}$. Then the semi-trajectory $\{(v, \kappa) \mid s \geq 0\}$ of $(v, \kappa)$ intersects $\{(v, \kappa) \mid v<0, v \kappa-\omega=0\}$ at $\left(v\left(s_{1}\right), \kappa\left(s_{1}\right)\right)$ for some $s_{1}>0$ such that it goes into the region $\mathcal{B}_{2}$ after $s_{1}$ and $(v(s), \kappa(s)) \rightarrow(-\infty, 0)$ as $s \rightarrow+\infty$. More precisely, $v^{\prime}<0$ and $\kappa^{\prime}<0$ on $\left(0, s_{1}\right), v^{\prime}<0$ and $\kappa^{\prime}>0$ on $\left(s_{1},+\infty\right)$, and $(v(s), \kappa(s)) \rightarrow$ $(-\infty, 0)$ as $s \rightarrow+\infty$. 
Proof. Note that $v^{\prime}(s)<0$ and $\kappa^{\prime}(s)<0$ for sufficiently small positive $s$. Suppose that the semi-trajectory $\mathcal{C}_{+}:=\{(v, \kappa) \mid s \geq 0\}$ of $(v, \kappa)$ does not enter $\mathcal{B}_{2}$. Then $\mathcal{C}_{+}$stays in $\mathcal{B}_{1}$ for all $s \geq 0$. Therefore, by Lemma 2.1 , we have

$$
\kappa(s) \leq \kappa(\delta)<0 \text { and } v^{\prime}(s) \leq \kappa(\delta)(1-\kappa(\delta))<0
$$

for all $s \geq \delta$ for an arbitrarily fixed $\delta>0$. From this it follows that $v(s) \rightarrow-\infty$ as $s \rightarrow+\infty$. This contradicts $v(s) \kappa(s)<\omega$ for all $s>0$. Thus $\mathcal{C}_{+}$shall intersect $\{(v, \kappa) \mid v<0, v \kappa-\omega=0\}$ at $\left(v\left(s_{1}\right), \kappa\left(s_{1}\right)\right)$ for some $s_{1}>0$ and then stay in $\mathcal{B}_{2}$ on $\left(s_{1},+\infty\right)$, by Lemma 2.1 .

Therefore, by Lemma 2.1 again, $v^{\prime}(s)<0$ and $\kappa^{\prime}(s)>0$ for all $s>s_{1}$. Thus we have $v(s) \rightarrow-\infty$ and $\kappa(s) \rightarrow c$ as $s \rightarrow+\infty$ for some $c \leq 0$. We claim that $c=0$. If $c<0$, then $\kappa^{\prime}(s) \rightarrow+\infty$ as $s \rightarrow+\infty$ and hence we reach a contradiction. Thus $c=0$ and the lemma is proved.

In the sequel, except otherwise stated, the trajectory of $(v, \kappa)$ is referred to the semi-trajectory $\mathcal{C}_{+}$of $(v, \kappa)$ as above.

Lemma 2.3. Let $(v, \kappa)$ be a solution of (2.1)-(2.2) with $\kappa(0)>0$. Then the following statements hold.

(1) If $\left(v\left(s_{0}\right), \kappa\left(s_{0}\right)\right) \in \mathcal{D}_{1}$ for some $s_{0} \geq 0$, then there exists $s_{1}>s_{0}$ such that the trajectory of $(v(s), \kappa(s))$ intersects $\{v>\omega, \kappa=1\}$ at $\left(v\left(s_{1}\right), \kappa\left(s_{1}\right)\right)$.

(2) If $\left(v\left(s_{0}\right), \kappa\left(s_{0}\right)\right) \in \mathcal{D}_{2}$ for some $s_{0} \geq 0$, then there exists $s_{1}>s_{0}$ such that the trajectory of $(v(s), \kappa(s))$ intersects $\{v \kappa-\omega=0, \kappa>1\}$ at $\left(v\left(s_{1}\right), \kappa\left(s_{1}\right)\right)$.

(3) If $\left(v\left(s_{0}\right), \kappa\left(s_{0}\right)\right) \in \mathcal{D}_{3}$ for some $s_{0} \geq 0$, then there exists $s_{1}>s_{0}$ such that the trajectory of $(v(s), \kappa(s))$ intersects $\{v<\omega, \kappa=1\}$ at $\left(v\left(s_{1}\right), \kappa\left(s_{1}\right)\right)$.

(4) If $\left(v\left(s_{0}\right), \kappa\left(s_{0}\right)\right) \in \mathcal{D}_{4}$ for some $s_{0} \geq 0$, then either there exists $s_{1}>s_{0}$ such that the trajectory of $(v(s), \kappa(s))$ hits $\{\kappa=0\}$ at $\left(v\left(s_{1}\right), \kappa\left(s_{1}\right)\right)$, or intersects $\{v \kappa-\omega=0,0<\kappa<1\}$ at $\left(v\left(s_{1}\right), \kappa\left(s_{1}\right)\right)$, or stays in the region $\mathcal{D}_{4}$ on $\left(s_{0},+\infty\right)$.

Proof. The lemma can be proved in a similar way to that in the proof of Lemma 2.2 by using the phase plane analysis.

LEMMA 2.4. Let $(v, \kappa)$ be the solution of (2.1)-(2.2) with the initial value $(0, b)$ for $b>0$. If $(v(s), \kappa(s))$ stays in the region $\{\kappa>0\}$ for all $s \geq 0$, then the trajectory of $(v, \kappa)$ can go around $(\omega, 1)$ only finitely many times. Moreover, it stays in $\mathcal{D}_{4}$ on $\left(s_{0},+\infty\right)$ for some $s_{0}>0$ and $\lim _{s \rightarrow+\infty}(v(s), \kappa(s))=(+\infty, 0)$.

Proof. First, we claim that the trajectory of any solution $(v(s), \kappa(s))$ can go around $(\omega, 1)$ only finitely many times. We fix $s_{0}>0$ satisfying $r\left(s_{0}\right)>1$. Since $r(s)>r\left(s_{0}\right)$ for $s>s_{0}$ and the circle $\left\{(v, \kappa) \mid(v-\omega)^{2}+(\kappa-1)^{2}=r\left(s_{0}\right)^{2}\right\}$ intersects the line $\{\kappa=0\}$, we have $\theta(s) \leq \theta\left(s_{0}\right)+2 \pi$ for $s>s_{0}$. On the other hand, from (2.4) it follows that $\theta^{\prime}(s) \leq 1+\omega / 2+r\left(s_{0}\right)$ on $\left[0, s_{0}\right]$, integrating the inequality from 0 to $s \leq s_{0}$, we obtain $\theta(s) \leq \theta(0)+\left(1+\omega / 2+r\left(s_{0}\right)\right) s_{0}$ for $0 \leq s \leq s_{0}$. Thus the trajectory of $(v(s), \kappa(s))$ goes around $(\omega, 1)$ only finitely many times.

Moreover, by Lemma $2.3,(v, \kappa)$ shall stay in the region $\mathcal{D}_{4}$ on $\left(s_{0},+\infty\right)$ for 
some $s_{0} \geq 0$. Noting that $v^{\prime}>0$ and $\kappa^{\prime}<0$ in $\mathcal{D}_{4}$ and that there is no equilibrium point of (2.1)-(2.2) in this region, we have $v \rightarrow \infty$ and $\kappa \rightarrow c$ as $s \rightarrow \infty$ for some $c \geq 0$. By a similar argument to that in the proof of Lemma 2.2, we have $c=0$. This completes the proof of this lemma.

With these lemmas, we can classify the solutions of $\left(P_{\omega}\right)$ into the following two types (see Figures 2 and 3).

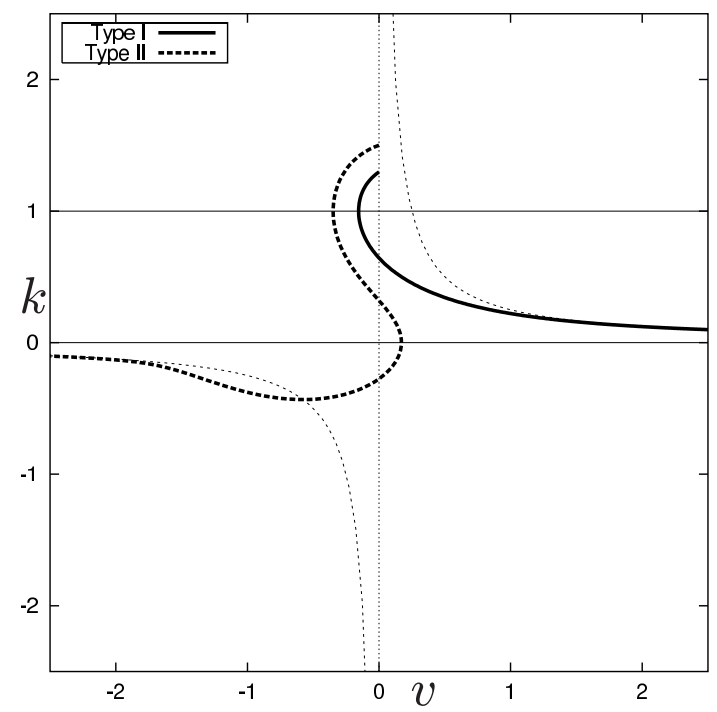

Fig. 2. The semi-trajectory of a type I solution (solid curve) and that of a type II solution (dashed curve) for $\omega=0.250$.

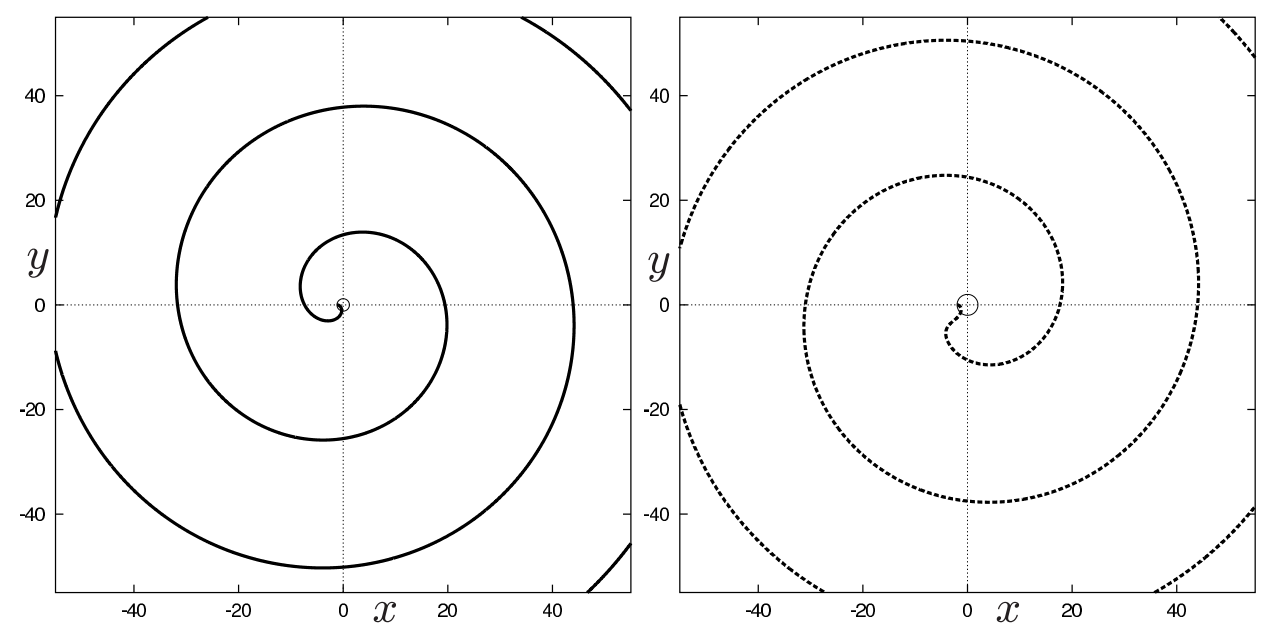

Fig. 3. Spiral curves which correspond to the type I solution (left) and the type II solution (right) in Figure 2. The center circles denote the core of the spirals. 


\section{DEFINITION 1.}

(I) The solution $(v(s), \kappa(s))$ of $\left(P_{\omega}\right)$ stays in the region $\{\kappa>0\}$ for all $s \geq 0$, and $\lim _{s \rightarrow+\infty}(v(s), \kappa(s))=(+\infty, 0)$.

(II) There exists $s_{0} \geq 0$ such that the solution $(v(s), \kappa(s))$ of $\left(P_{\omega}\right)$ stays in the region $\{\kappa<0\}$ for all $s \in\left(s_{0},+\infty\right)$. Moreover, we have $\lim _{s \rightarrow+\infty}(v(s), \kappa(s))=$ $(-\infty, 0)$.

Though trajectories of $\left(P_{\omega}\right)$ for different $\omega$ may intersect, the next lemma shows that they do not intersect for a while.

Lemma 2.5. Let $0<\omega_{1}<\omega_{2}$ and let $\left(v_{i}(s), \kappa_{i}(s)\right)$ be the solution of (2.1)(2.2) for $\omega=\omega_{i}$ with the initial value $\left(v_{i}(0), \kappa_{i}(0)\right)=\left(p_{i}, 1\right)$ and $p_{i}<\omega_{i}(i=1,2)$. Define

$$
\begin{aligned}
& \sigma_{i}^{-}=\inf \left\{s<0 \mid \kappa_{i}>1 \text { on }(s, 0)\right\} \\
& \sigma_{i}^{+}=\sup \left\{s>0 \mid 0<\kappa_{i}<1 \text { on }(0, s)\right\}
\end{aligned}
$$

and

$$
\begin{aligned}
& \gamma_{i}^{-}=\left\{\left(v_{i}(s), \kappa_{i}(s)\right) \mid \sigma_{i}^{-}<s<0\right\} \\
& \gamma_{i}^{+}=\left\{\left(v_{i}(s), \kappa_{i}(s)\right) \mid 0<s<\sigma_{i}^{+}\right\},
\end{aligned}
$$

for $i=1,2$. If $p_{2} \leq p_{1}<\omega_{1}$, then the following statements hold:

(i) $\gamma_{1}^{-} \cap \gamma_{2}^{-}=\emptyset$ and $\gamma_{1}^{+} \cap \gamma_{2}^{+}=\emptyset$.

(ii) Both $\sigma_{1}^{-}$and $\sigma_{2}^{-}$are finite, and $v_{1}\left(\sigma_{1}^{-}\right)<v_{2}\left(\sigma_{2}^{-}\right)$.

(iii) If both $\sigma_{1}^{+}$and $\sigma_{2}^{+}$are finite and if $\kappa_{1}\left(\sigma_{1}^{+}\right)=\kappa_{2}\left(\sigma_{2}^{+}\right):=\kappa_{0} \in\{0,1\}$, then we have $v_{1}\left(\sigma_{1}^{+}\right)<v_{2}\left(\sigma_{2}^{+}\right)$when $\kappa_{0}=1, v_{1}\left(\sigma_{1}^{+}\right)>v_{2}\left(\sigma_{2}^{+}\right)$when $\kappa_{0}=0$.

Proof. (i) We only prove $\gamma_{1}^{-} \cap \gamma_{2}^{-}=\emptyset$, since the other statement can be treated similarly. Since $v_{i}^{\prime}(s)<0$ on $\left(\sigma_{i}^{-}, 0\right)$ for $i=1$, 2 , we can view $\kappa_{1}$ and $\kappa_{2}$ as functions of $v$. If $p_{2}<p_{1}$, then $\kappa_{1}(v)<\kappa_{2}(v)$ in a right neighborhood of $p_{1}$. This also holds for the case $p_{2}=p_{1}:=p$, since

$$
\frac{d v_{1}}{d \kappa_{1}}=0=\frac{d v_{2}}{d \kappa_{2}}, \quad \frac{d^{2} v_{1}}{d \kappa_{1}^{2}}=-\frac{1}{p-\omega_{1}}>-\frac{1}{p-\omega_{2}}=\frac{d^{2} v_{2}}{d \kappa_{2}^{2}}>0 \quad \text { at }(p, 1) .
$$

Suppose that $\gamma_{1}^{-} \cap \gamma_{2}^{-} \neq \emptyset$. Then there exists some $q>p_{1}$ satisfying $\kappa_{1}<\kappa_{2}$ on $\left(p_{1}, q\right)$ and $\kappa_{1}(q)=\kappa_{2}(q):=k>1$. This contradicts

$$
\frac{d \kappa_{1}}{d v}(q)=\frac{q k-\omega_{1}}{k(1-k)}<\frac{q k-\omega_{2}}{k(1-k)}=\frac{d \kappa_{2}}{d v}(q)
$$

Therefore, $\gamma_{1}^{-} \cap \gamma_{2}^{-}=\emptyset$.

(ii) By the phase plane analysis, it is clear that $\sigma_{1}^{-}$and $\sigma_{2}^{-}$are finite. Moreover, the statement (i) implies $v_{1}\left(\sigma_{1}^{-}\right) \leq v_{2}\left(\sigma_{2}^{-}\right)$. Suppose that $v_{1}\left(\sigma_{1}^{-}\right)=v_{2}\left(\sigma_{2}^{-}\right):=$ $q$. Then we have $q>\omega_{2}, \kappa_{1}<\kappa_{2}$ on $\left(p_{1}, q\right)$ and $\kappa_{1}\left(q^{-}\right)=\kappa_{2}\left(q^{-}\right)=1$. These 
contradict

$$
\frac{d v_{1}}{d \kappa_{1}}=0=\frac{d v_{2}}{d \kappa_{2}}, \quad 0>\frac{d^{2} v_{1}}{d \kappa_{1}^{2}}=-\frac{1}{q-\omega_{1}}>-\frac{1}{q-\omega_{2}}=\frac{d^{2} v_{2}}{d \kappa_{2}^{2}} \quad \text { at }(q, 1) .
$$

Thus we have $v_{1}\left(\sigma_{1}^{-}\right)<v_{2}\left(\sigma_{2}^{-}\right)$.

(iii) By (i), $v_{1}\left(\sigma_{1}^{+}\right) \leq v_{2}\left(\sigma_{2}^{+}\right)$, if $\kappa_{0}=1$; and $v_{1}\left(\sigma_{1}^{+}\right) \geq v_{2}\left(\sigma_{2}^{+}\right)$, if $\kappa_{0}=0$. Suppose that $v_{1}\left(\sigma_{1}^{+}\right)=v_{2}\left(\sigma_{2}^{+}\right):=q$. Then we have $q>\omega_{2}$, if $\kappa_{0}=1, \kappa_{1}>\kappa_{2}$ on $\left(p_{1}, q\right)$, and $\kappa_{1}\left(q^{-}\right)=\kappa_{2}\left(q^{-}\right)=\kappa_{0}$. These also contradict $(2.5)$ or

$$
\frac{d v_{1}}{d \kappa_{1}}=0=\frac{d v_{2}}{d \kappa_{2}}, \quad \frac{d^{2} v_{1}}{d \kappa_{1}^{2}}=-\frac{1}{\omega_{1}}<-\frac{1}{\omega_{2}}=\frac{d^{2} v_{2}}{d \kappa_{2}^{2}}<0 \quad \text { at }(q, 0) .
$$

Thus the lemma is proved.

\section{Rotating Spirals with Positive Curvature}

In this section, we shall study the behavior of the solution of (2.1)-(2.2) with the initial value $(0,1)$ in order to show the existence of type I solutions for sufficiently small $\omega$. Let $\left(v_{\omega}(s), \kappa_{\omega}(s)\right)$ be the solution of $\left(P_{\omega}\right)$ with $b=1$ and let $\left(r_{\omega}(s), \theta_{\omega}(s)\right)$ be the corresponding solution of $(2.3)-(2.4)$ with the initial value $(\omega, \pi)$ throughout the remainder of this section. Set

$$
\begin{aligned}
& A_{1}=\left\{\omega>0 \mid \kappa_{\omega}^{\prime}(s) \text { reaches zero before } \kappa_{\omega}(s) \text { does }\right\}, \\
& A_{2}=\left\{\omega>0 \mid \kappa_{\omega}(s) \text { reaches zero before } \kappa_{\omega}^{\prime}(s) \text { does }\right\} .
\end{aligned}
$$

Note that $A_{1}$ and $A_{2}$ are open sets with $A_{1} \cap A_{2}=\emptyset$. Moreover, Lemma 2.5 implies that $A_{1}$ and $A_{2}$ are connected. Since the set $\left\{(v-\omega)^{2}+(\kappa-1)^{2} \geq 1\right\}$ is positively invariant, it is easy to see that $[1,+\infty) \subset A_{2}$.

We shall claim that $\left(0, \omega_{0}\right) \subset A_{1}$ for some positive constant $\omega_{0}$.

Lemma 3.1. The set $A_{1}$ contains $\left(0, \omega_{0}\right)$ for some small positive constant $\omega_{0}$. Moreover, the trajectory of $(2.1)-(2.2)$ with the initial value $(0,1)$ goes around $(\omega, 1)$ as many times as we want, if $\omega$ is small enough.

Proof. Let $\omega \in(0,1 / 2)$. Since $r_{\omega}^{\prime}(s) \leq \omega r_{\omega}(s)$, we have $r_{\omega}(s) \leq 1 / 2$ for $s \in\left[0, s_{0}\right]$, where $s_{0}:=-\ln (2 \omega) / \omega$. This means that $\kappa_{\omega}(s)>0$ for all $s \in\left[0, s_{0}\right]$. From this and (2.4) it follows that

$$
\theta_{\omega}^{\prime}(s) \geq 1-\omega / 2-r_{\omega}(s) \geq(1-\omega) / 2
$$

on $\left[0, s_{0}\right]$. Integrating this inequality from 0 to $s_{0}$, we obtain

$$
\theta_{\omega}\left(s_{0}\right)-\theta_{\omega}(0) \geq \frac{1}{2}(1-\omega) s_{0}=\frac{1}{2}\left(\frac{1}{\omega}-1\right) \ln \frac{1}{2 \omega} .
$$

This implies that $\theta_{\omega}\left(s_{0}\right)-\theta_{\omega}(0) \geq \pi$ for all $\omega \in\left(0, \omega_{0}\right)$, where $\omega_{0}$ is the unique positive constant satisfying $\omega<1 / 2$ and

$$
\left(\frac{1}{\omega}-1\right) \ln \frac{1}{2 \omega}=2 \pi \text {. }
$$


Hence the trajectory must intersect $\{v \kappa=\omega, \kappa>0\}$. Since the right-hand side of (3.3) tends to $+\infty$ as $\omega \rightarrow 0$, there exists a trajectory of (2.1)-(2.2) with the initial value $(0,1)$ which goes around $(\omega, 1)$ as many times as we want, if $\omega$ is small enough. The lemma is proved.

Let $\omega_{*}=\sup A_{1}$ and $\omega^{*}=\inf A_{2}$. Then $0<\omega_{*} \leq \omega^{*}<1$ and $\omega_{*}, \omega^{*} \notin A_{1} \cup A_{2}$. Moreover, for any $\omega \in\left[\omega_{*}, \omega^{*}\right]$, the solution $\left(v_{\omega}(s), \kappa_{\omega}(s)\right)$ satisfies that $v^{\prime}>0$ and $\kappa^{\prime}<0$ for all $s \geq 0$.

We shall claim that $\omega_{*}=\omega^{*}$. Suppose $\omega_{*}<\omega^{*}$. Set $\left(v_{1}(s), \kappa_{1}(s)\right)=$ $\left(v_{\omega_{*}}(s), \kappa_{\omega_{*}}(s)\right)$ and $\left(v_{2}(s), \kappa_{2}(s)\right)=\left(v_{\omega^{*}}(s), \kappa_{\omega^{*}}(s)\right)$. Since $v_{i}^{\prime}(s)>0$ for $s>0$ for $i=1,2$, we can view $\kappa_{1}$ and $\kappa_{2}$ as functions of $v$. It follows from Lemma 2.5 that $\kappa_{1}>\kappa_{2}$ on $(0,+\infty)$. Since $\kappa_{i} \rightarrow 0$ as $s \rightarrow+\infty$ for $i=1$, 2 , we can choose sufficiently large $v_{0}$ such that $0<\kappa_{2}<\kappa_{1}<1 / 2$ for all $v \geq v_{0}$. Then

$$
\frac{d\left(\kappa_{1}-\kappa_{2}\right)}{d v}=v\left(\frac{1}{1-\kappa_{1}}-\frac{1}{1-\kappa_{2}}\right)+\left(\frac{\omega^{*}}{\kappa_{2}\left(1-\kappa_{2}\right)}-\frac{\omega_{*}}{\kappa_{1}\left(1-\kappa_{1}\right)}\right)>0,
$$

for $v \geq v_{0}$. Integrating this inequality from $v_{0}$ to $+\infty$, we obtain

$$
\left(\kappa_{1}-\kappa_{2}\right)(+\infty)-\left(\kappa_{1}-\kappa_{2}\right)\left(v_{0}\right)>0,
$$

contradicting $\kappa_{1}\left(v_{0}\right)>\kappa_{2}\left(v_{0}\right)$. Hence we conclude that $\omega_{*}=\omega^{*}$. Hereafter we write $\bar{\omega}=\omega_{*}\left(=\omega^{*}\right)$.

Lemma 3.2. For any fixed $\omega>0$, the problem $\left(P_{\omega}\right)$ has at most one type I solution up to translation.

Proof. We assume that $\left(v_{1}, \kappa_{1}\right)$ and $\left(v_{2}, \kappa_{2}\right)$ are type I solutions of $\left(P_{\omega}\right)$ whose trajectories do not coincide. Then we can view $\kappa_{1}$ and $\kappa_{2}$ as functions of $v$ on $(0,+\infty)$. Without loss of generality, we may assume $1 / 2>\kappa_{1}>\kappa_{2}$ on $\left(v_{0},+\infty\right)$ for some $v_{0}>0$. Then, letting $\omega_{*}=\omega^{*}=\omega$ in $(3.4)$, we obtain $\left(\kappa_{1}-\kappa_{2}\right)^{\prime}(v)>0$ for sufficiently large $v$. This leads to a contradiction in the same way as above and the proof is completed.

THEOREM 1. There is a type I solution of $\left(P_{\omega}\right)$ if and only if $\omega \in(0, \bar{\omega}]$.

Proof. In the proof of this theorem, we let the solution $(r(s ; \omega, b), \theta(s ; \omega, b))$ of $(2.3)-(2.4)$ correspond to the solution $(v(s ; \omega, b), \kappa(s ; \omega, b))$ of $\left(P_{\omega}\right)$.

First, we assume that $\omega>\bar{\omega}$. Then we have $v_{\omega}^{\prime}(s)>0, \kappa_{\omega}^{\prime}(s)<0$ for all $s \in\left(0, s_{1}\right)$ and $\kappa_{\omega}\left(s_{1}\right)=0$ for some $s_{1}>0$. From this and Lemma 2.3 it follows that any trajectory starting from $(0, b)$ with $b>0$ must intersect the $v$-axis. Hence $(v(s ; \omega, b), \kappa(s ; \omega, b))$ is of type II for $b>0$. Since $(v(s ; \omega, b), \kappa(s ; \omega, b))$ is also of type II for $b \leq 0$, there is no type I solution for $\omega>\bar{\omega}$.

Next, given a fixed $\omega<\bar{\omega}$, we consider the set

$$
A:=\left\{b \in(0,1] \mid \kappa_{b}(s) \text { reaches zero before } \kappa_{b}^{\prime}(s) \text { does }\right\} .
$$

By the definition of $\bar{\omega}, 1 \notin A$. Note that $\bar{\omega}<1$. On the other hand, $b \in A$ for $b \leq 1-\sqrt{1-\omega^{2}}$, since $r(0 ; \omega, b)^{2}=(1-b)^{2}+\omega^{2} \geq 1$ and $r(s ; \omega, b)$ is increasing. 
Therefore the supremum $b_{0}$ of $A$ exists. Moreover, it is easy to see that the solution $\left(v\left(s ; \omega, b_{0}\right), \kappa\left(s ; \omega, b_{0}\right)\right)$ starting from the point $\left(0, b_{0}\right)$ is of type I with $v^{\prime}\left(s ; \omega, b_{0}\right)>0$ and $\kappa^{\prime}\left(s ; \omega, b_{0}\right)<0$ for all $s>0$. Hence we have at least one type I solution of $\left(P_{\omega}\right)$ for $\omega \in(0, \bar{\omega})$. The theorem is proved.

Next we want to count exactly the number of type I solutions of $\left(P_{\omega}\right)$ for each given rotation frequency $\omega$. For any $\omega>0$, we set

$$
R_{\omega}(t)=\frac{1}{\omega} r_{\omega}\left(\frac{t}{\omega}\right), \quad \vartheta_{\omega}(t)=\theta_{\omega}\left(\frac{t}{\omega}\right) .
$$

Then $(R(t), \vartheta(t))=\left(R_{\omega}(t), \vartheta_{\omega}(t)\right)$ satisfies the system

$$
\begin{aligned}
& \frac{d R}{d t}=R \sin ^{2} \vartheta \\
& \frac{d \vartheta}{d t}=\frac{1}{\omega}+\sin \vartheta \cos \vartheta+R \sin \vartheta,
\end{aligned}
$$

with the initial condition $(R(0), \vartheta(0))=(1, \pi)$.

Note that if $(v, \kappa)$ is of type I, then $(R, \vartheta) \rightarrow(+\infty, 2 m \pi)$ as $t \rightarrow+\infty$ for some $m \in \mathbf{N}$; and if $(v, \kappa)$ is of type II, then $(R, \vartheta) \rightarrow(+\infty,(2 m-1) \pi)$ as $t \rightarrow+\infty$ for some $m \in \mathbf{N}$.

Also, note that if $\vartheta\left(t_{0}\right)=m \pi$ for some $t_{0} \geq 0$ and $m \in \mathbf{N}$, then we can view $R$ as a function of $\vartheta$ for sufficiently small $|\vartheta-m \pi|$. Moreover, we have

$$
\left.\frac{d R}{d \vartheta}\right|_{\vartheta=m \pi}=\left.\frac{d^{2} R}{d \vartheta^{2}}\right|_{\vartheta=m \pi}=0 \text { and }\left.\frac{d^{3} R}{d \vartheta^{3}}\right|_{\vartheta=m \pi}=2 \omega R\left(t_{0}\right)>0 .
$$

Hence we can also view $\vartheta$ as a function of $R$ near $R\left(t_{0}\right)$. On the other hand, for $\vartheta \neq m \pi$, from (3.5) it follows that we can view $\vartheta$ as a function of $R$ and that $\vartheta(R)$ satisfies the following equation

$$
\frac{d \vartheta}{d R}=\frac{(1 / \omega)+\sin \vartheta \cos \vartheta+R \sin \vartheta}{R \sin ^{2} \vartheta} \text { when } \vartheta \neq m \pi \text { for any } m \in \mathbf{N}
$$

Therefore, we can view $\vartheta$ as a function of $R$ for all $R \in[1, \infty)$.

LEMMA 3.3. Let $\left(R_{i}(t), \vartheta_{i}(t)\right)=\left(R_{\omega_{i}}(t), \vartheta_{\omega_{i}}(t)\right)$ for $i=1,2$.

(1) If $\omega_{1}<\omega_{2}$, then $\vartheta_{1}(R)>\vartheta_{2}(R)$ for all $R>1$.

(2) If $\lim _{t \rightarrow+\infty}\left(R_{1}(t), \vartheta_{1}(t)\right)=\lim _{t \rightarrow \infty}\left(R_{2}(t), \vartheta_{2}(t)\right)=(+\infty, 2 m \pi)$ for some $m \in \mathbf{N}$, then $\omega_{1}=\omega_{2}$.

Proof. (1) By (3.6) we have $\vartheta_{1}(R)>\vartheta_{2}(R)$ for $R>1$ with $R-1$ sufficiently small. Furthermore, from (3.8) it follows that $\vartheta_{1}(R)>\vartheta_{2}(R)$ for all $R$ with $\vartheta_{i}(R) \in$ $(\pi, 2 \pi), i=1,2$. Using this argument, we can show that $\vartheta_{1}(R)>\vartheta_{2}(R)$ for all $R \in\left(0, R_{0}\right)$ where $R_{0}$ satisfies $\vartheta_{1}\left(R_{0}\right)=\vartheta_{2}(\infty)$. If $R_{0}=\infty$, we are done. Otherwise, from (3.7) it follows that $\vartheta_{1}(R)>\vartheta_{2}(\infty)$ for all $R>R_{0}$. Hence $\vartheta_{1}(R)>\vartheta_{2}(R)$ for all $R>1$. This completes the proof. 
(2) For contradiction, we assume that $\omega_{1}<\omega_{2}$. Note that $\vartheta_{i}(t)<2 m \pi$, $i=1,2$, for all $t \geq 0$ by Lemma 2.4. Since we can view $\vartheta_{i}$ as a function of $R$, $i=1,2$, we have $\vartheta_{2}(R)<\vartheta_{1}(R)<2 m \pi$ for all $R>1$ and $\lim _{R \rightarrow+\infty} \vartheta_{1}(R)=$ $\lim _{R \rightarrow+\infty} \vartheta_{2}(R)=2 m \pi$. On the other hand, $d^{2} \vartheta_{i} / d t^{2}=R \sin ^{3} \vartheta_{i}<0$ whenever $d \vartheta_{i} / d t=0$ for $i=1,2$ and $R$ is sufficiently large. Therefore, $d \vartheta_{i} / d t>0, i=1,2$, for all $t>t_{0}$ for some $t_{0}>0$. Thus we have

$$
\begin{aligned}
\frac{d \vartheta_{1}}{d R} & =\frac{\left(1 / \omega_{1}\right)+\sin \vartheta_{1} \cos \vartheta_{1}+R \sin \vartheta_{1}}{R \sin ^{2} \vartheta_{1}} \\
& >\frac{\left(1 / \omega_{2}\right)+\sin \vartheta_{2} \cos \vartheta_{2}+R \sin \vartheta_{2}}{R \sin ^{2} \vartheta_{2}}=\frac{d \vartheta_{2}}{d R}
\end{aligned}
$$

for all sufficiently large $R$. This contradicts the fact that $\lim _{R \rightarrow+\infty}\left(\vartheta_{1}(R)-\right.$ $\left.\vartheta_{2}(R)\right)=0$. The proof is completed.

The next corollary follows from the above lemma.

Corollary 3.4. Suppose $0<\omega_{1}<\omega_{2}$. Let $\left(v_{i}, \kappa_{i}\right)=\left(v_{\omega_{i}}, \kappa_{\omega_{i}}\right)$ and $\left(r_{i}, \theta_{i}\right)=$ $\left(r_{\omega_{i}}, \theta_{\omega_{i}}\right)$ for $i=1,2$. If there exist positive constants $s_{1}$ and $s_{2}$ satisfying $\theta_{1}\left(s_{1}\right)=$ $\theta_{2}\left(s_{2}\right)=(2 m+1) \pi$ for some $m \in \mathbf{N}$, then $v_{2}\left(s_{2}\right)<v_{1}\left(s_{1}\right)<0$.

Proof. Let $\left(R_{i}, \vartheta_{i}\right)=\left(R_{\omega_{i}}, \vartheta_{\omega_{i}}\right)$ and set $t_{i}=\omega_{i} s_{i}$ for $i=1,2$. From the fact $R_{i}(0)=1$ and (3.5), it follows that $R_{i}(t)>1$ for all $t>0$. By the assumption $\vartheta_{\omega_{1}}\left(t_{1}\right)=\vartheta_{\omega_{2}}\left(t_{2}\right)$ and the part (1) of Lemma 3.3, we can conclude that $1<R_{1}\left(t_{1}\right)<$ $R_{2}\left(t_{2}\right)$. Recall that $v_{i}=\omega_{i}+r_{i} \cos \theta_{i}, i=1,2$. Hence we have

$$
\begin{aligned}
v_{2}\left(s_{2}\right)-v_{1}\left(s_{1}\right) & =\left(\omega_{2}+r_{2}\left(s_{2}\right) \cos \theta_{2}\left(s_{2}\right)\right)-\left(\omega_{1}+r_{1}\left(s_{1}\right) \cos \theta_{1}\left(s_{1}\right)\right) \\
& =\left(\omega_{2}-r_{2}\left(s_{2}\right)\right)-\left(\omega_{1}-r_{1}\left(s_{1}\right)\right) \\
& =\omega_{2}\left(1-R_{2}\left(t_{2}\right)\right)-\omega_{1}\left(1-R_{1}\left(t_{1}\right)\right) \\
& <\omega_{2}\left(1-R_{2}\left(t_{2}\right)\right)-\omega_{2}\left(1-R_{1}\left(t_{1}\right)\right) \\
& =\omega_{2}\left(R_{1}\left(t_{1}\right)-R_{2}\left(t_{2}\right)\right)<0 .
\end{aligned}
$$

Combining this with the fact that $r_{i}$ is increasing $(i=1,2)$, we obtain $v_{2}\left(s_{2}\right)<$ $v_{1}\left(s_{1}\right)<0$. The proof is completed.

THEOREM 2. There exists a sequence of positive numbers

$$
\bar{\omega}=\omega_{0}>\omega_{1}>\omega_{2}>\cdots>\omega_{m}>\cdots>0
$$

with $\omega_{m} \rightarrow 0$ as $m \rightarrow \infty$ such that $\left(P_{\omega}\right)$ has exactly $2 m$ type I solutions, if $\omega \in$ $\left(\omega_{m}, \omega_{m-1}\right)$; and $\left(P_{\omega}\right)$ has exactly $2 m+1$ type I solutions, if $\omega=\omega_{m}$.

Proof. First, we note that $\left(v_{\bar{\omega}}, \kappa_{\bar{\omega}}\right)$ is the only type I solution of $\left(P_{\bar{\omega}}\right)$ by Lemma 3.2.

By the definition of $\bar{\omega}$ and the continuous dependence of $\left(P_{\omega}\right)$ in $\omega$, for any $\omega \in(0, \bar{\omega})$ sufficiently close to $\bar{\omega}$, there exists $s_{0}>0$ such that the trajectory of 
$\left(v_{\omega}(s), \kappa_{\omega}(s)\right)$ intersects $\{v \kappa-\omega=0, \kappa>0\}$ at $s=s_{0}$ and that $r_{\omega}\left(s_{0}\right)>1$, $\theta_{\omega}\left(s_{0}\right) \in(\pi, 2 \pi)$. Hence, using Lemma 2.3 and noting that $r_{\omega}$ is increasing, we have $\kappa_{\omega}\left(s_{1}\right)=0, \theta_{\omega}\left(s_{1}\right) \in(3 \pi, 4 \pi)$ for some $s_{1}>s_{0}$ and $\left(v_{\omega}(s), \kappa_{\omega}(s)\right) \rightarrow(-\infty, 0)$ as $s \rightarrow+\infty$ (see Figure 4 ).

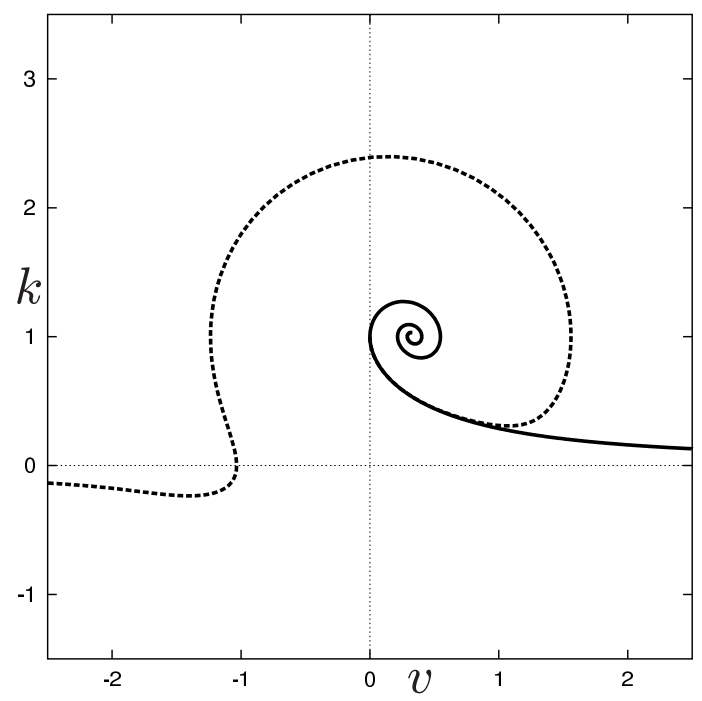

Fig. 4. The trajectory of $\left(v_{\bar{\omega}}, \kappa_{\bar{\omega}}\right)$ (solid curve) and the semi-trajectory of $\left(v_{\omega}, \kappa_{\omega}\right)$ with $\omega \in(0, \bar{\omega})$ sufficiently close to $\bar{\omega}$ (dashed curve).

Define $B_{1}$ to be the set of all positive numbers $\omega \in(0, \bar{\omega})$ such that $\kappa_{\omega}\left(s_{1}\right)=0$ and $\theta_{\omega}\left(s_{1}\right) \in(3 \pi, 4 \pi)$ for some $s_{1}>0$. It follows from Lemma 2.5, Lemma 2.3 and Corollary 3.4 that if $\hat{\omega} \in B_{1}$, then $\omega \in B_{1}$ for all $\omega \in[\hat{\omega}, \bar{\omega})$. Moreover, by Lemma 3.1 and the above discussion, the set $B_{1}$ is nonempty and bounded from below. Therefore, $\omega_{1}:=\inf B_{1}$ exists. Furthermore, from part (2) of Lemma 3.3 it follows that $\omega_{1}$ is the unique among all positive numbers $\omega$ such that $\theta_{\omega}(s) \rightarrow 4 \pi$ as $s \rightarrow+\infty$.

By an inductive argument, we can find a sequence of positive numbers

$$
\bar{\omega}=\omega_{0}>\omega_{1}>\omega_{2}>\cdots>\omega_{m}>\cdots>0
$$

such that

(1) If $\omega \in\left(\omega_{m}, \omega_{m-1}\right)$, then $\kappa_{\omega}\left(s_{0}\right)=0$ and $\theta_{\omega}\left(s_{0}\right) \in((2 m+1) \pi, 2(m+1) \pi)$ for some $s_{0}>0$. Moreover, the trajectory of $\left(v_{\omega}, \kappa_{\omega}\right)$ hits the line $\{v=0, \kappa>0\}$ exactly $2 m$ or $2 m+1$ times and $\left(v_{\omega}(s), \kappa_{\omega}(s)\right) \rightarrow(-\infty, 0)$ as $s \rightarrow+\infty$.

(2) If $\omega=\omega_{m}$, then $\theta_{\omega_{m}}(s) \rightarrow 2(m+1) \pi$ as $s \rightarrow+\infty$. Moreover, the trajectory of $\left(v_{\omega_{m}}, \kappa_{\omega_{m}}\right)$ hits the line $\{v=0, \kappa>0\}$ exactly $2 m+1$ times and $\left(v_{\omega_{m}}(s), \kappa_{\omega_{m}}(s)\right) \rightarrow(+\infty, 0)$ as $s \rightarrow+\infty$. 
Now, we turn to the problem $\left(P_{\omega}\right)$ for a fixed $\omega \in\left[\omega_{m}, \omega_{m-1}\right)$. Firstly, we suppose that $\omega \in\left(\omega_{m}, \omega_{m-1}\right)$. Let $\left(0, \kappa_{i}\right)(i=1, \ldots, 2 m)$ be the $i$ th intersection point of the trajectory of $\left(v_{\omega}, \kappa_{\omega}\right)$ with the line $\{v=0, \kappa>0\}$ such that

$$
\kappa_{2 m-1}>\cdots>\kappa_{1}>1=\kappa_{0}>\kappa_{2}>\cdots>\kappa_{2 m-2}>\kappa_{2 m} .
$$

Here we define $\kappa_{2 m}=0$ in the case where the trajectory of $\left(v_{\omega}, \kappa_{\omega}\right)$ intersects $\{v=0, \kappa>0\}$ exactly $2 m$ times. Consider the set

$$
\left\{\alpha \in\left(\kappa_{2 m}, \kappa_{2 m-2}\right) \mid \kappa(s ; \omega, \alpha) \text { reaches zero before } \kappa^{\prime}(s ; \omega, \alpha) \text { does }\right\} .
$$

Then by the same argument as in the proof of Theorem 1 , we see that the supremum $\alpha_{0}$ of this set has the property that $\left(v\left(s ; \omega, \alpha_{0}\right), \kappa\left(s ; \omega, \alpha_{0}\right)\right)$ is of type I. Furthermore, the trajectory $\left\{\left(v\left(s ; \omega, \alpha_{0}\right), \kappa\left(s ; \omega, \alpha_{0}\right)\right) \mid s \in \mathbf{R}\right\}$ intersects the line $\{v=0, \kappa>0\}$ at exactly $2 m$ points. Therefore, from Lemma 3.2 it follows that we have exactly $2 m$ distinct solutions of $\left(P_{\omega}\right)$ such that they are all of type I and they share the same trajectory $\left\{\left(v\left(s ; \omega, \alpha_{0}\right), \kappa\left(s ; \omega, \alpha_{0}\right)\right) \mid s \in \mathbf{R}\right\}$.

For the case $\omega=\omega_{m}$, it follows that the trajectory $\left\{\left(v_{\omega_{m}}(s), \kappa_{\omega_{m}}(s)\right) \mid s \in \mathbf{R}\right\}$ intersects the line $\{v=0, \kappa>0\}$ at exactly $2 m+1$ points. Therefore, from Lemma 3.2 it follows that we have exactly $2 m+1$ distinct solutions of $\left(P_{\omega}\right)$ such that they are all of type I and they share the same trajectory $\left\{\left(v_{\omega_{m}}(s), \kappa_{\omega_{m}}(s)\right) \mid s \in \mathbf{R}\right\}$.

Finally, we remark that $\omega_{m} \rightarrow 0$ as $m \rightarrow \infty$ by Lemmas 2.4 and 3.1.

\section{Classification of Rotating Spirals}

Recall the problem $\left(P_{\omega}\right)$ :

$$
\begin{aligned}
& \frac{d v}{d s}=\kappa(1-\kappa), \\
& \frac{d \kappa}{d s}=v \kappa-\omega, \\
& v(0)=0, \quad \kappa(0)=b .
\end{aligned}
$$

By Lemma 2.1, for each $\omega>0$, there exist $\kappa^{*}=\kappa^{*}(\omega)>1$ and $s^{*}=s^{*}(\omega)>0$ such that the solution $(v, \kappa)$ of $\left(P_{\omega}\right)$ with $b=\kappa^{*}$ satisfies $(v, \kappa) \in\{v<0, \kappa>0\}$ on $\left(0, s^{*}\right)$ and $\left(v\left(s^{*}\right), \kappa\left(s^{*}\right)\right)=(0,0)$.

We introduce the following types of spiral solutions.

DeFinition 2.

(A) $(v, \kappa)$ stays in the region $\{v<0, \kappa<0\}$ on $(0, \infty)$ and $(v(s), \kappa(s)) \rightarrow(-\infty, 0)$ as $s \rightarrow+\infty$.

(B) There exist $s_{1}>0$ and $s_{2}>0$ such that $(v, \kappa)$ stays in the region $\{v>0, \kappa>$ $0\}$ on $\left(0, s_{1}\right),\{v>0, \kappa<0\}$ on $\left(s_{1}, s_{2}\right),\{v<0, \kappa<0\}$ on $\left(s_{2}, \infty\right)$, and $(v(s), \kappa(s)) \rightarrow(-\infty, 0)$ as $s \rightarrow+\infty$.

(C) There exist $s_{1}>0, s_{2}>0$ and $s_{3}>0$ such that $(v, \kappa)$ stays in the region $\{v<0, \kappa>0\}$ on $\left(0, s_{1}\right),\{v>0, \kappa>0\}$ on $\left(s_{1}, s_{2}\right),\{v>0, \kappa<0\}$ on $\left(s_{2}, s_{3}\right),\{v<0, \kappa<0\}$ on $\left(s_{3}, \infty\right)$, and $(v(s), \kappa(s)) \rightarrow(-\infty, 0)$ as $s \rightarrow+\infty$. 
(D) There exists $s_{1}>0$ such that $(v, \kappa)$ stays in the region $\{v<0, \kappa>0\}$ on $\left(0, s_{1}\right),\{v<0, \kappa<0\}$ on $\left(s_{1}, \infty\right)$, and $(v(s), \kappa(s)) \rightarrow(-\infty, 0)$ as $s \rightarrow+\infty$.

\subsection{The case for $\omega>\bar{\omega}$}

Theorem 3. Given $\omega>\bar{\omega}$. Let $\kappa_{*}=\kappa_{*}(\omega)<0$ be such that the solution of $\left(P_{\omega}\right)$ with $b=1$ intersects $\{v=0, \kappa<0\}$ at $\left(0, \kappa_{*}\right)$. Then the following statements hold.

(1) If $b \leq 0$, then $(v, \kappa)$ is of type $(\mathrm{A})$.

(2) If $b \in(0,1]$, then $(v, \kappa)$ is of type $(\mathrm{B})$.

(3) If $b \in\left(1, \kappa^{*}\right)$, then $(v, \kappa)$ is of type $(\mathrm{C})$.

(4) If $b \geq \kappa^{*}$, then $(v, \kappa)$ is of type (D).

(5) For each $b \in\left(1, \kappa^{*}\right)$, there exist unique $\tilde{b} \in(0,1)$ and $\hat{b} \in\left(\kappa_{*}, 0\right)$ such that the solution $(v, \kappa)$ of $\left(P_{\omega}\right)$ passes through $(0, \tilde{b})$ and $(0, \hat{b})$.

Proof. Using the argument of Theorem 1 , for any $b \in(0,1]$ there exists $s_{1}>0$ such that $v^{\prime}>0$ and $\kappa^{\prime}<0$ on $\left[0, s_{1}\right)$, and $\kappa\left(s_{1}\right)=0$. Moreover $\kappa_{*}$ is well-defined. Thus $(v, \kappa)$ is of type $(\mathrm{B})$ for any $b \in(0,1]$. The remaining statements immediately follow from the phase plane analysis.

4.2. The case for $\omega \in(0, \bar{\omega}]$

4.2.1. The case for $\omega=\omega_{m}$

Given $\omega=\omega_{m}$. Then by Theorem 2 there exists a sequence of positive numbers

$$
b_{m}>\cdots>b_{1}>1>a_{1}>\cdots>a_{m}
$$

such that $\left(P_{\omega}\right)$ has exactly $2 m+1$ type I solutions with the initial values $\left(0, b_{i}\right)$, $\left(0, a_{i}\right), i=1, \ldots, m$, and $(0,1)$, respectively, if $m \geq 1$. Hereafter we set $a_{0}=b_{0}=1$.

THEOREM 4. Given $\omega=\omega_{m}$. Then the following statements hold.

(1) If $b \leq 0$, then $(v, \kappa)$ is of type $(\mathrm{A})$.

(2) If $b \in\left(0, a_{m}\right)$, then $(v, \kappa)$ is of type $(\mathrm{B})$.

(3) If $b \in\left(b_{m}, \kappa^{*}\right)$, then $(v, \kappa)$ is of type $(\mathrm{C})$.

(4) If $b \geq \kappa^{*}$, then $(v, \kappa)$ is of type (D).

(5) For each $b \in\left(b_{m}, \kappa^{*}\right)$, there exists a unique $\tilde{b} \in\left(0, a_{m}\right)$ and $\hat{b} \in(-\infty, 0)$ such that the solution $(v, \kappa)$ of $\left(P_{\omega}\right)$ passes through $(0, \tilde{b})$ and $(0, \hat{b})$.

Proof. The theorem follows from the definition of $\omega_{m}$ and the phase plane analysis.

For $m \geq 1$, if we continue backward the trajectory with initial value $(0, b)$ for any $b>b_{m}$, then we can obtain the solution of $\left(P_{\omega}\right)$ with $b \in\left(a_{m}, b_{m}\right) \backslash$ $\left\{a_{m-1}, \ldots, 1, \ldots, b_{m-1}\right\}$. In other words, any solution of $\left(P_{\omega}\right)$ with $b \in\left(a_{m}, b_{m}\right) \backslash$ $\left\{a_{m-1}, \ldots, 1, \ldots, b_{m-1}\right\}$ shall reach $(0, \hat{b})$ at some $s>0$ for some $\hat{b}>b_{m}$.

More precisely, let $\gamma$ be the (whole) trajectory of the solution of $\left(P_{\omega}\right)$ which passes through $\left(0, \kappa^{*}\right)$. Then $\gamma$ intersects $\left\{v=0, a_{m}<\kappa<b_{m}\right\}$ at $\left(0, c_{i}\right),\left(0, c_{i}^{\prime}\right)$, 
$i=0,1, \ldots, m-1$, with $c_{i} \in\left(a_{i+1}, a_{i}\right)$ and $c_{i}^{\prime} \in\left(b_{i}, b_{i+1}\right)$. Hence we have

$$
b_{m}>c_{m-1}^{\prime}>\cdots>b_{1}>c_{0}^{\prime}>b_{0}=1=a_{0}>c_{0}>a_{1}>\cdots>c_{m-1}>a_{m} .
$$

Moreover, for each $i=1, \ldots, m$, if $b \in\left(c_{i-1}^{\prime}, b_{i}\right)$, then the trajectory of $(v, \kappa)$ goes around $(\omega, 1)$ counterclockwise exactly $m-i+1$ times and hits the positive $v$-axis firstly at $(0, \tilde{b})$ for some $\tilde{b} \in\left(a_{i}, c_{i-1}\right)$ and lastly at $(0, \hat{b})$ for some $\hat{b} \in\left(\kappa^{*}, \infty\right)$; while if $b \in\left(b_{i-1}, c_{i-1}^{\prime}\right)$, then the trajectory goes around $(\omega, 1)$ counterclockwise $m-i+1$ times and hits the positive $v$-axis first at $(0, \tilde{b})$ for some $\tilde{b} \in\left(c_{i-1}, a_{i-1}\right)$ and last at $(0, \hat{b})$ for some $\hat{b} \in\left(0, a_{m}\right)$.

Indeed, for any $b \in\left(c_{0}^{\prime}, b_{1}\right)$ the trajectory of the solution of $\left(P_{\omega}\right)$ passes through a unique point $\left(0, \tilde{b}_{i}\right)$ with $\tilde{b}_{i} \in\left(a_{i}, c_{i-1}\right)$ and a unique point $\left(0, \hat{b}_{i}\right)$ with $\hat{b}_{i} \in$ $\left(c_{i}^{\prime}, b_{i+1}\right)$ for each $i=1, \ldots, m$, where $c_{m}^{\prime}:=\kappa^{*}$ and $b_{m+1}:=\infty$. Similarly, for any $b \in\left(b_{0}, c_{0}^{\prime}\right)$ the trajectory of the solution of $\left(P_{\omega}\right)$ passes through a unique point $\left(0, \tilde{b}_{i}\right)$ with $\tilde{b}_{i} \in\left(c_{i-1}, a_{i-1}\right)$ for each $i=1, \ldots, m+1$ and a unique point $\left(0, \hat{b}_{i}\right)$ with $\hat{b}_{i} \in\left(b_{i}, c_{i}^{\prime}\right)$ for each $i=1, \ldots, m$, where $c_{m}:=0$ and $c_{m}^{\prime}:=\kappa^{*}$. Moreover, this correspondence is one-to-one and onto.

\subsubsection{The case for $\omega \in\left(\omega_{m}, \omega_{m-1}\right)$}

Given $\omega \in\left(\omega_{m}, \omega_{m-1}\right)$. Then by Theorem 2 there exists a sequence of positive numbers

$$
b_{m}>\cdots>b_{1}>1>a_{1}>\cdots>a_{m}
$$

such that $\left(P_{\omega}\right)$ has exactly $2 m$ type I solutions with initial values $\left(0, b_{i}\right)$ and $\left(0, a_{i}\right)$ for $i=1, \ldots, m$, respectively.

TheOREM 5. Given $\omega \in\left(\omega_{m}, \omega_{m-1}\right)$. Then the following statements hold.

(1) If $b \leq 0$, then $(v, \kappa)$ is of type (A).

(2) If $b \in\left(0, a_{m}\right)$, then $(v, \kappa)$ is of type $(\mathrm{B})$.

(3) If $b \in\left(b_{m}, \kappa^{*}\right)$, then $(v, \kappa)$ is of type $(\mathrm{C})$.

(4) If $b \geq \kappa^{*}$, then $(v, \kappa)$ is of type (D).

(5) For each $b \in\left(b_{m}, \kappa^{*}\right)$, there exists a unique $\tilde{b} \in\left(0, a_{m}\right)$ and $\hat{b} \in(-\infty, 0)$ such that the solution $(v, \kappa)$ of $\left(P_{\omega}\right)$ passes through $(0, \tilde{b})$ and $(0, \hat{b})$.

Proof. Again, the theorem follows from the definition of $\omega_{m}$ and the phase plane analysis.

Similar to the case for $\omega=\omega_{m}$, we can easily described the solutions of $\left(P_{\omega}\right)$ for $b \in\left(a_{m}, b_{m}\right)$. This can be done by dividing it into two cases depending on whether $(0,1)$ lies on the (backward) trajectory $\gamma$ for the solution of $\left(P_{\omega}\right)$ with initial value $\left(0, \kappa^{*}\right)$. Since this can be easily seen by the phase plane analysis, we omit the details.

\section{Geometric Explanations}

We study the existence of steadily rotating spiral waves from the kinematic model equation. This is equivalent to find a planar curve such that its tip rotates 
along a circle with a constant angular frequency $\omega>0$. We restrict ourselves to the case when the tip is rotating along a circle in the counterclockwise direction. Without loss of generality we take the normal velocity $u=1-\kappa$. Since the tangential velocity $G(t)$ at the tip is identically equal to zero, the normal vector at the tip is perpendicular to the position vector of the tip by taking the center of the core circle to be the origin. But, the tangent vector is pointed inward to the center if $\kappa(0)>1$; and is pointed outward to the center if $\kappa(0)<1$. Also, we recall that the radius of core circle is given by $\rho=|1-\kappa(0)| / \omega$.

We consider both constant-sign and sign-changing solutions by studying a system of two first order equations $\left(P_{\omega}\right)$. We choose the sign of curvature to be positive if the curve is right-winding or equivalently winding in the clockwise direction. It is shown that the curvature function can change sign at most once. Moreover, spirals with positive curvature can exist if and only if $\omega \in(0, \bar{\omega}]$ for some positive $\bar{\omega}$. This fact was proved already in [2] and [3]. Here we provide an alternative proof.

In general, there is no restriction on $\omega$, if we do not restrict ourselves to spirals with positive curvature. In fact, there is a continuum of spirals for any given angular frequency $\omega>0$ as shown in $\S 4$.

We give some geometrical explanations of some solutions which we obtained in this paper as follows. Define $\kappa_{*}:=-\infty$ for $\omega \in(0, \bar{\omega}]$ and $a_{m}=b_{m}=1$ for $\omega \geq \bar{\omega}$.

(i) There is a unique circle (core) such that the tip tangent is pointed inward and the curve is first right-winding and then left-winding, if $\kappa(0)>\kappa^{*}$.
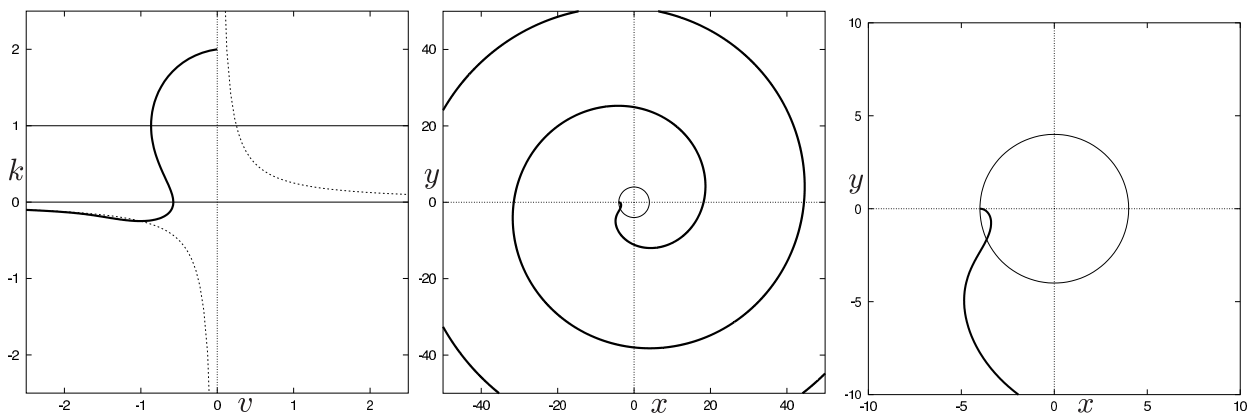

Fig. 5. (left) The semi-trajectory of the solution of $\left(P_{\omega}\right)$ with $\kappa(0)>\kappa^{*}$ for $\omega=0.250$, which corresponds to Case (i) in Section 5. (middle) The corresponding spiral and the core. (right) The magnification of the near core region.

(ii) There are three circles (they may be coincide) with the center at the origin such that the tip tangent is pointed inward, the curve is perpendicular to these circles at the intersection points with the tangents at the second and the third circle being pointed outward, and the curvature changes sign between the second and the third circles, if $\kappa(0) \in\left(b_{m}, \kappa^{*}\right)$. In fact, let $s_{1}>0$ and $s_{2}>s_{1}$ be such that the curve intersects with the second circle and the third one at $s_{1}$ and $s_{2}$, respectively. Then $\kappa\left(s_{1}\right) \in\left(0, a_{m}\right)$ and $\kappa\left(s_{2}\right) \in\left(\kappa_{*}, 0\right)$. This gives 
another two spirals with tip curvatures in $\left(0, a_{m}\right)$ and $\left(\kappa_{*}, 0\right)$, respectively, since the system is autonomous.

(iii) There is a unique circle (core) such that the tip tangent is pointed outward and the curve is left-winding (with negative curvature), if $\kappa(0)<\kappa_{*}$ (in the case when $\omega>\bar{\omega})$.

(iv) The spiral with $\kappa(0)=0$ is a part of the one with $\kappa(0)=\kappa^{*}$; and the spiral with $\kappa(0)=\kappa_{*}($ in the case when $\omega>\bar{\omega})$ is a part of the one with $\kappa(0)=1$.

(v) There are two circles (may be coincide) such that the tip tangent is pointed inward, the curve is perpendicular to these two circles at the intersection points with the tangent at the intersection point of the curve and the second circle being pointed outward, and the curvature of the curve is positive everywhere, if $\kappa(0)=b_{m}$ (in the case when $\left.\omega \in(0, \bar{\omega}]\right)$. In fact, the curvature at the intersection point of the curve and the second circle is $a_{m}$. This also gives
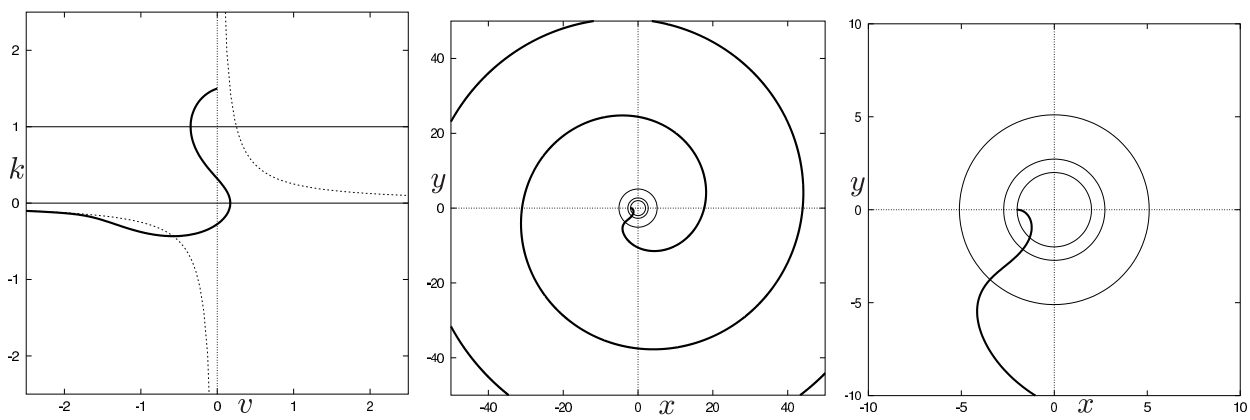

Fig. 6. (left) The semi-trajectory of the solution of $\left(P_{\omega}\right)$ with $\kappa(0) \in\left(b_{m}, \kappa^{*}\right)$ for $\omega=0.250$, which corresponds to Case (ii) in Section 5. (middle) The corresponding spiral and the cores. (right) The magnification of the near core region.
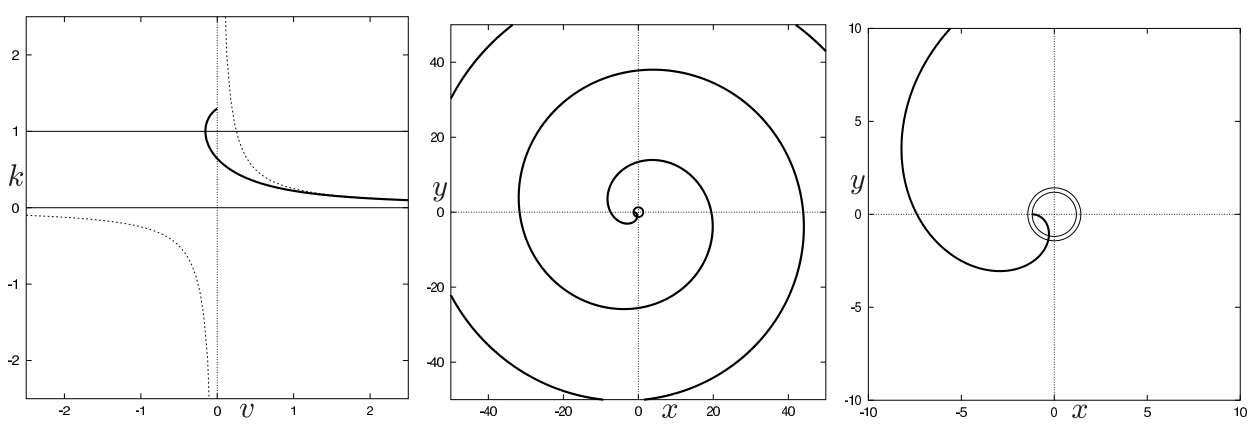

Fig. 7. (left) The semi-trajectory of the solution of $\left(P_{\omega}\right)$ with $\kappa(0)=b_{m}$ for $\omega=0.250$, which corresponds to Case (v) in Section 5. (middle) The corresponding spiral and the cores. (right) The magnification of the near core region. 
another spiral with tip curvature being equal to $a_{m}$ such that the curvature of the curve is positive everywhere.

All the curves described as above have no self-intersections (see Figures 5, 6 and 7$)$. For $\omega \in(0, \bar{\omega}]$, we are not sure whether the corresponding curves to the solutions of $\left(P_{\omega}\right)$ with $b \in\left(a_{m}, b_{m}\right)$ have self-intersections. We suspect that the curve has self-intersection(s) for any solution $(v, \kappa)$ such that $v$ has more than two zeros with $\kappa \geq 0$. But, this interesting question is still left for open.

Acknowledgments. This work was partially supported by the National Science Council of the Republic of China under the grant NSC 92-2115-M-003-008 and NSC 94-2115-M-019-002. This work was also partially supported by the National Center for Theoretic Sciences at Taipei during a visit of the second and third authors to Taiwan in September, 2004. The authors would like to thank the referees for many helpful comments on the presentation of this paper.

\section{References}

[1] P.K. Brazhnik, Exact solutions for the kinematic model of autowaves in two-dimensional excitable media. Physica D, 94 (1996), 205-220.

[ 2 ] B. Fiedler, J.-S. Guo and J.-C. Tsai, Multiplicity of rotating spirals under curvature flows with normal tip motion. J. Diff. Equations, 205 (2004), 211-228.

[ 3 ] B. Fiedler, J.-S. Guo and J.-C. Tsai, Rotating spirals of curvature flows: a center manifold approach. Annali di Matematica Pura ed Applicata (to appear).

[ 4 ] J.-S. Guo, N. Ishimura and C.-C. Wu, Self-similar solutions for the kinematic model equation of spiral waves. Physica D, 198 (2004), 197-211.

[ 5 ] J.-S. Guo, C.-P. Lo and J.-C. Tsai, The structure of solutions for equations related to the motions of plane curves. ANZIAM J., 45 (2004), 585-592.

[6] R. Ikota, N. Ishimura and T. Yamaguchi, On the structure of steady solutions for the kinematic model of spiral waves in excitable media. Japan J. Indust. Appl. Math., 15 (1998), 317-330.

[ 7 ] J.P. Keener, The core of the spiral. SIAM J. Appl. Math., 52 (1992), 1370-1390.

[ 8 ] E. Meron, Pattern formation in excitable media. Physics Reports (Review Section of Physics Letters), 218 (1992), 1-66.

[9] A.S. Mikhailov, V.A. Davydov and A.S. Zykov, Complex dynamics of spiral waves and motion of curves. Physica D, 70 (1994), 1-39.

[10] T. Ogiwara and K.-I. Nakamura, Spiral traveling wave solutions of some parabolic equations on annuli. Josai Mathematical Monographs, 2 (2000), 15-34.

[11] J.J. Tyson and J.P. Keener, Singular perturbation theory of traveling waves in excitable media (a review). Physica D, 32 (1988), 327-361.

[12] V.S. Zykov, Simulation of Wave Process in Excitable Media. Manchester University Press, 1984 . 